\title{
Prospective Randomized Controlled Study Comparing Primary Surgery Versus Neoadjuvant Chemotherapy Followed by Surgery in Gastric Carcinoma
}

\author{
Ramachandra $^{1} \cdot$ Vipin Goel $^{1} \cdot$ Kvvn Raju $^{1} \cdot$ T. Subramanyeshwar Rao ${ }^{1} \cdot$ Patnaik $^{1} \cdot$ Nusrath $^{1} \cdot$ Santa $^{2} \cdot$ Sudha $^{\text {Murthy }}{ }^{3}$
}

Received: 21 January 2019 / Accepted: 7 March 2019 / Published online: 22 March 2019

(C) Indian Association of Surgical Oncology 2019

\begin{abstract}
Neoadjuvant chemotherapy in gastric cancer can treat micro metastatic disease and can increase the resectability rate. The trial was to compare early outcomes after primary surgery versus neoadjuvant chemotherapy followed by surgery in gastric adenocarcinoma. The primary aim of the study was to compare resectability and R 0 resection rates in upfront surgery $\mathrm{v} / \mathrm{s}$ chemotherapy followed by surgery arm. A secondary aim was to see if neoadjuvant chemotherapy is well tolerated or not by comparing postoperative morbidity and mortality. The study consisted of 60 consecutive patients of carcinoma stomach randomized into primary surgery and neoadjuvant chemotherapy followed by surgery arms. Morbidity, pathological status, and mortality data were collected and analyzed. Mean operating time in primary surgery arm was $290 \pm 46.5 \mathrm{~min}$, while in NACTarm, it was 316.7 $\pm 56.6 \mathrm{~min}$, respectively. When postop complications were compared between the arms, it was not significant. Comparing the histopathological report of two groups, there was no significant difference between differentiated, $T$ stage, mean lymph node harvest, R0 resection, PNI, and LVI. Neoadjuvant chemotherapy showed a trend towards improving in the R0 resection rate. There is no increase in postoperative morbidity and mortality with neoadjuvant chemotherapy.
\end{abstract}

Keywords Primary surgery $\cdot$ Neoadjuvant chemotherapy $\cdot$ Gastric carcinoma

\section{Introduction}

Gastric cancer is one of the leading causes of cancer in India [1]. About $90 \%$ of stomach tumors are adenocarcinoma. The 5 -year survival is less than $30 \%$ in developed countries and around $20 \%$ in developing countries [2]. The outcome among patients with gastric cancer is determined by the stage of the disease at presentation. Surgery remains the main modality of treatment for stomach cancer. Despite radical surgery, many patients relapse with local and systemic recurrence, which leads to poor OS. Adjuvant chemotherapy may improve

Vipin Goel

vipinrgoel@gmail.com

1 Department of Surgical Oncology, Basavatarakam Indo American Hospital, Road no 10, Banjara Hills, Hyderabad, India

2 Department of Medical Oncology, Basavatarakam Indo American Hospital, Road no 10, Banjara Hills, Hyderabad, India

3 Department of Pathology, Basavatarakam Indo American Hospital, Road no 10, Banjara Hills, Hyderabad, India survival but, the morbidity of surgery may limit the ability to deliver postoperative chemotherapy effectively [3]. Therefore, there is a lot of interest nowadays in neoadjuvant or preoperative chemotherapy. Neoadjuvant chemotherapy can treat micro metastatic disease and neoadjuvant chemotherapy in gastric cancer offers an opportunity to deliver systemic treatment when the patient is best able to tolerate it, since initiating postoperative chemotherapy is usually delayed following gastric cancer surgery [4]. To see if neoadjuvant chemo increases the resectability rate after neoadjuvant chemotherapy, a prospective randomized study was conducted in the Department of Surgical Oncology of Basavatarakam Indo American Cancer Hospital and Research Institute, Hyderabad, to compare early outcomes after primary surgery versus neoadjuvant chemotherapy followed by surgery in gastric adenocarcinoma.

\section{Aim}

The primary aim of the study was to compare resectability and $\mathrm{R} 0$ resection rates in upfront surgery $\mathrm{v} / \mathrm{s}$ chemotherapy 
followed by surgery arm. A secondary aim was to see if neoadjuvant chemotherapy is well tolerated or not by comparing postoperative morbidity and mortality.

\section{Methods}

The study was conducted in the Department of Surgical Oncology in Basavatarakam Indo American Cancer and Research Institute, Hyderabad, between 1/5/2014 and 31/12/ 2015. The study consisted of 60 consecutive patients of carcinoma stomach irrespective of age and sex with histologically proven resectable adenocarcinoma of the stomach in patients with ECOG performance status 0-2. Patients were excluded if they present with distant metastasis or gastric outlet obstruction. Patients with serious medical condition or major systemic diseases which make unfit for major surgery were also excluded.

Patients were randomized into two groups of 30 patients each in primary surgery and neoadjuvant chemotherapy followed by surgery arms. After a routine work up and staging evaluation, random number was generated by the random sequence generator at Random.org. All even number patients were allotted to primary surgery arm and all odd number patients were allocated to neoadjuvant chemotherapy followed by surgery arm. Preoperatively, all patients were investigated in the same manner with routine blood investigations, including blood counts, liver and renal functions, an electrocardiogram, and an endoscopy with biopsy. Computed tomography (CT) of the abdomen and pelvis was done. In surgery arm, patients underwent surgical resection within 14 days of randomization. Surgery was radical gastrectomy either total or subtotal gastrectomy. In radical total gastrectomy, the whole stomach was removed, with the proximal line of division through the distal esophagus, and the distal line of division through the proximal duodenum. The resection also included the greater and lesser omentum and any other organ involved by growth. The procedure for a radical subtotal distal gastrectomy was the same, but a small, viable gastric remnant was left intact. The resection line was at least $5 \mathrm{~cm}$ proximally and at least $1 \mathrm{~cm}$ distally from the edge of the macroscopic tumor. All patients underwent spleen and pancreas preserving D2 lymph node dissection. In neoadjuvant chemotherapy arm, patients received preoperative chemotherapy with IV Cisplatin $\left(70 \mathrm{mg} / \mathrm{m}^{2}\right)$ on day 1 along with 5-Fluorouracil $800 \mathrm{mg} / \mathrm{m}^{2} /$ day as an IV infusion on day $1-3$, repeated every 21 days for 3 cycles in the absence of disease progression or unacceptable toxicity. Surgery was performed 4 to 6 weeks after day 1 of the last course of chemotherapy. Surgery technique and level of resection was like arm I only.

During surgery, operative time, blood loss (estimated by the volume of suction and the weight of gauze), and the amount of blood transfusion were recorded. Postoperative complications were graded as described by Dindo et al. Postoperative complications were categorized as surgical and nonsurgical complications, occurring during the hospital stay, and abscesses needing drainage, intra-abdominal or anastomotic bleeding needing transfusion or re-exploration, ileus, delayed gastric emptying as described by Wente et al., lymphatic leakage, and anastomotic leakage. Nonsurgical complications were cardiac, pulmonary, urinary, renal, and hepatic complications. Mortality was defined as any death occurring within 30 days of surgery. The depth of tumor invasion, tumor size, margins, the number of harvested lymph nodes (HLNs), and positive lymph nodes were determined by pathological analysis. Histological staging was classified according to the 7th edition of the American Joint Committee on Cancer Staging Manual.

\section{Statistical methods}

Continuous data were expressed as mean \pm standard deviation (SD). Comparisons of categorical and continuous variables were performed using the Fisher exact test and paired $t$ test, respectively. A $p$ value of less than 0.05 was considered statistically significant. All statistical analyses were performed using statistical software Graph Pad Prism version 6 for Windows.

\section{Results}

In the present study, 80 cases were assessed for eligibility; out of 80 patients, 20 were excluded and 30 patients randomized to primary surgery arm and 30 patients to neoadjuvant chemotherapy followed by surgery. Out of whom, 24 patients from primary surgery arm and 27 patients from neoadjuvant chemotherapy arm underwent surgery. In 6 of $30(20 \%)$ and 3 of $30(10 \%)$ patients were unresectable in primary surgery and NACT arms, respectively. Un-resectability in both arm patients was due to detection of metastatic disease at the time of surgery (Fig. 1).

In the total study population, 67\% (40) were male and 33\% (20) were female patients. In the primary surgery arm, $60 \%$ (18) were male and $40 \%$ (12) were female, while in NACT arm, $73 \%$ (22) were male and $27 \%$ (8) were female patients. The mean age in primary surgery arm was $51.83 \pm 9.78$ years and mean age in NACT arm was $50.73 \pm 9.54$ years. Mean BMI was $21.81 \pm 4.86$ and $21.5 \pm 3.03$ in primary surgery and NACT arms, respectively. In the primary surgery arm, $79 \%$ (19) of patients underwent distal radical gastrectomy, while $21 \%$ underwent total radical gastrectomy. In NACT arm, $74 \%$ (20) underwent distal radical gastrectomy, while $26 \%$ (7) underwent total radical gastrectomy (Fig. 2).

Mean blood loss, mean ICU stay, mean time for flatus passage, mean time for starting oral feeding, and mean 
Fig. 1 Study overview

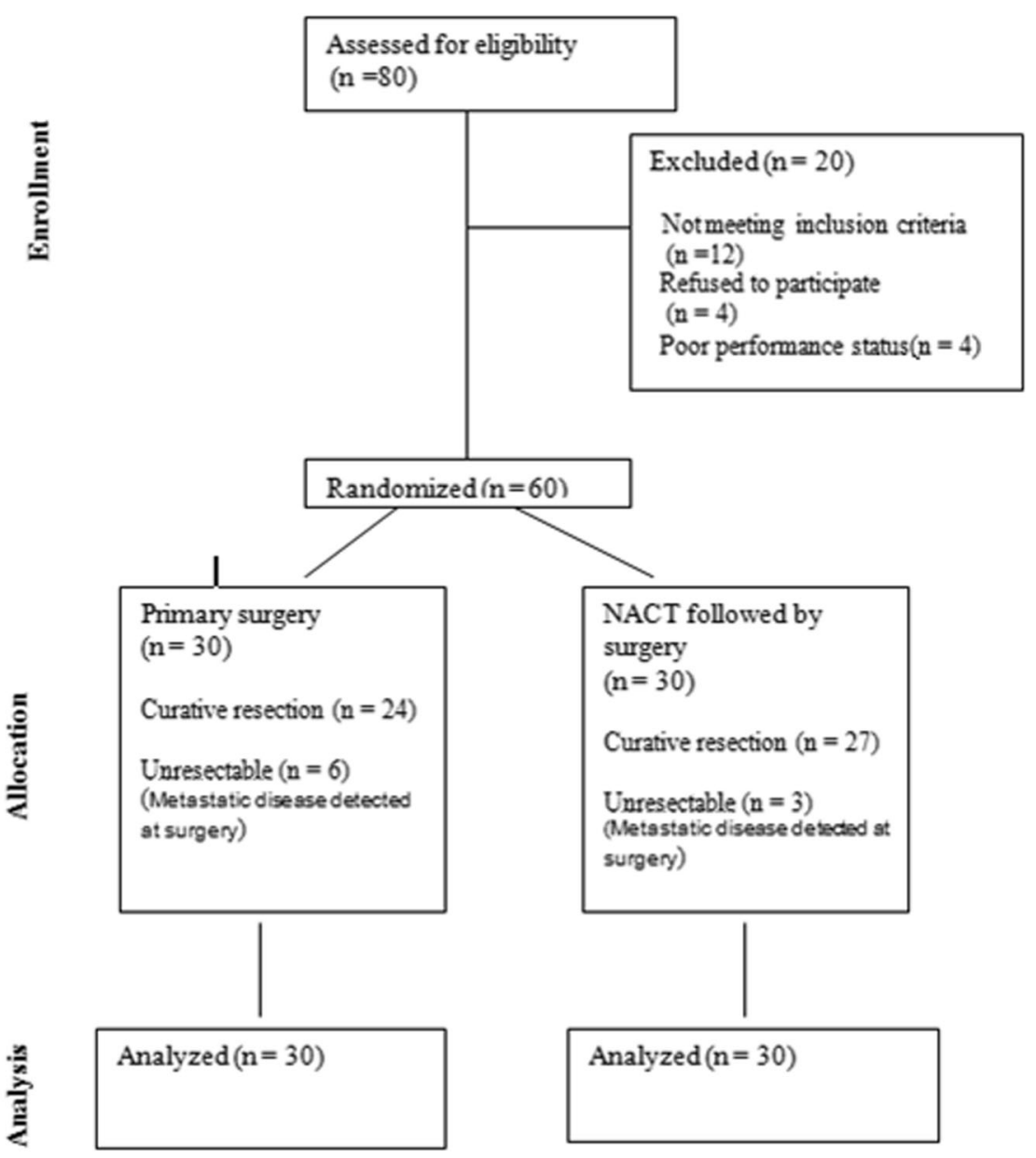

hospital stay were almost equal in both groups. There was no significant difference between two groups. Mean operating time in primary surgery arm was $290 \pm 46.5 \mathrm{~min}$, while in NACT arm, it was $316.7 \pm 56.6 \mathrm{~min}$, respectively. There was

Fig. 2 Patient characteristics

\begin{tabular}{|l|l|l|}
\hline CHARACTERISTIC & $\begin{array}{l}\text { PRIMARY } \\
\text { SURGERY }\end{array}$ & NACT ARM \\
\hline MEAN AGE (YEARS) & $51.83 \pm 9.78$ & $50.73 \pm 9.54$ \\
\hline & & \\
\hline SEX & & $22(73 \%)$ \\
\hline MALE & $18(60 \%)$ & $8(27 \%)$ \\
\hline FEMALE & $12(40 \%)$ & $21.5 \pm 3.03$ \\
\hline & & $20(79 \%)$ \\
\hline MEAN BMI (KG/m2) & $21.8 \pm 4.86$ & $7(26 \%)$ \\
\hline $\begin{array}{l}\text { SURGERY } \\
\text { GASTALECTOMY }\end{array}$ & $19(79 \%)$ & \\
\hline $\begin{array}{l}\text { TOTAL } \\
\text { GASTRECTOMY }\end{array}$ & $5(21 \%)$ & \\
\hline
\end{tabular}


Fig. 3 Operative data

\begin{tabular}{|l|l|l|l|}
\hline \multicolumn{1}{|c|}{ CHARACTERISTIC } & \multicolumn{1}{c|}{ PRIMARY SURG } & \multicolumn{1}{c|}{$\begin{array}{c}\text { SURGEY AFTER } \\
\text { NACT }\end{array}$} & \multicolumn{1}{c|}{ p Value } \\
\hline $\begin{array}{l}\text { MEAN OPERATIV TIME } \\
\text { (MTS) }\end{array}$ & $290 \pm 46.5$ & $316.7 \pm 56.6$ & 0.08 \\
\hline MEAN BLOOD LOSS & $210.4 \pm 51.0$ & $225.9 \pm 71.2$ & 0.62 \\
\hline MEAN BLOOD TRANSFUSION & $0.42 \pm 0.82$ & $0.52 \pm 0.75$ & 0.43 \\
\hline $\begin{array}{l}\text { MEAN DAY OF PASSAGE OF } \\
\text { FIRST FLATUS }\end{array}$ & $3.3 \pm 0.5$ & $3.3 \pm 0.6$ & 0.7 \\
\hline $\begin{array}{l}\text { MEAN DAY OF } \\
\text { COMMENCEMENT OF ORAL } \\
\text { FEEDING }\end{array}$ & $5.8 \pm 3.1$ & $5.7 \pm 1.4$ & 0.29 \\
\hline $\begin{array}{l}\text { TYPE A DELAYED GASTRIC } \\
\text { EMPTYING (NO. OF } \\
\text { PATIENTS) }\end{array}$ & $6(25 \%)$ & $10(37 \%)$ & 0.38 \\
\hline MEAN HOSPITAL STAY & $8.6 \pm 1.7$ & $9.0 \pm 1.8$ & 0.32 \\
\hline
\end{tabular}

trend towards an increase in operating time post neoadjuvant chemotherapy (Fig. 3).

When comparing postoperative mortality and morbidity, there was no perioperative mortality in either of arms. Nine grade I events, 11 grade II events, and one grade III event occurred in primary surgery arm. In NACT arm, 18 grade I events, 14 grade II events, and no grade III events noted. Only one major complication occurred in the primary surgery arm with anastomotic leak and the intra-abdominal collection, which was drained under ultrasound guidance. When total numbers of patients with grade II or more complications were compared between the arms, it was not significant (Fig. 4).

Comparing the histopathological report of two groups, there was no significant difference between differentiated, $\mathrm{T}$ stage, mean lymph node harvest, R0 resection, PNI, and LVI. In NACT arm, $5(18.5 \%)$ patients had a pathological complete response. One striking difference was lower $\mathrm{N}$ stage after neoadjuvant chemotherapy with $p$ value of 0.08 . Tumor
Fig. 4 Postoperative complications

\begin{tabular}{|l|c|c|c|}
\hline \multicolumn{1}{|c|}{ MORBIDITY } & $\begin{array}{c}\text { PRIMARY } \\
\text { SURGERY }\end{array}$ & $\begin{array}{c}\text { SURGERY AFTER } \\
\text { NACT }\end{array}$ & p VALUE \\
\hline Grade I & 9 & 18 & \\
\hline Grade II & 11 & 14 & \\
\hline Grade III & 1 & 0 & \\
\hline $\begin{array}{l}\text { Total number of patients with Grade 2 } \\
\text { or more complication }\end{array}$ & 10 & 10 & 0.78 \\
\hline
\end{tabular}

\begin{tabular}{|l|c|c|}
\hline \multicolumn{1}{|c|}{ Surgical complications } & & NACT \\
\hline & Primary Surgery & 0 \\
\hline Bleeding & 0 & $10 / 27(37 \%)$ \\
\hline Transfusion & $6 / 24(25 \%)$ & 0 \\
\hline Anastamoticleak & $1 / 24(4 \%)$ & 0 \\
\hline Intra abdominal abscess & $1 / 24(4 \%)$ & 0 \\
\hline Fistula & 0 & $1 / 27(3.7 \%)$ \\
\hline Sepsis & $1 / 24(4 \%)$ & \\
\hline Wound infection & 0 & $11 / 27(40.7 \%)$ \\
\hline & & 0 \\
\hline \multicolumn{1}{|c|}{ Non surgical complication } & $8 / 24(33 \%)$ & $10 / 27(37 \%)$ \\
\hline Pulmonary & 0 & 0 \\
\hline Cardiac & $6 / 24(25 \%)$ & \\
\hline Delayed gastricemptying & $1 / 24(4 \%)$ & \\
\hline Others & & \\
\hline
\end{tabular}


regression grading was mild or no response (Gr. 3) in $37 \%$ (10) of patients, moderate (Gr. 2) in $26 \%$ (7), marked response (Gr. 1) in $18 \%$ (5) patients (Fig. 5).

\section{Discussion}

Mean operating time in primary surgery arm was $290 \pm$ $46.5 \mathrm{~min}$, while in NACT arm, it was $316.7 \pm 56.6 \mathrm{~min}$, respectively. There was a trend towards increased operative time in NACT arm probably due to more inflammatory response induced by chemotherapy.

In the present study, R0 resection rate was $87 \%$ and $96 \%$ in primary surgery and NACT arms, which did not reach statistical significance (Fisher's exact test, $p$ value $=0.33$, odds ratio $0.26 ; 95 \% \mathrm{CI}, 0.026$ to 2.78 ). In a MAGIC trial by Cunningham et al. [5], among patients treated by radical surgery, resection was considered curative by the operating surgeon in 169 of 213 patients $(79.3 \%)$ in the perioperative chemotherapy group as compared with 166 of 236 patients $(70.3 \%)$ in the surgery group $(p=0.03)$. In FNCLCC and FFCD Multicenter Phase III Trial, Y Chou et al. [6] reported that $\mathrm{R} 0$ resection rate was $84 \%$ in the perioperative chemotherapy group versus $74 \%$ in the surgery alone group which was statistically significant $(p=0.04)$. In another trial, Schumacher et al. [7] reported that $\mathrm{R} 0$ resection rates were $66.70 \%$ and $81.9 \%$ in surgery alone and perioperative chemotherapy arms, respectively which was statistically significant ( $p=0.03$ ). But in his meta-analysis of 4 RCTs, Xu A M et al. [8] had reported that neoadjuvant chemotherapy did not result

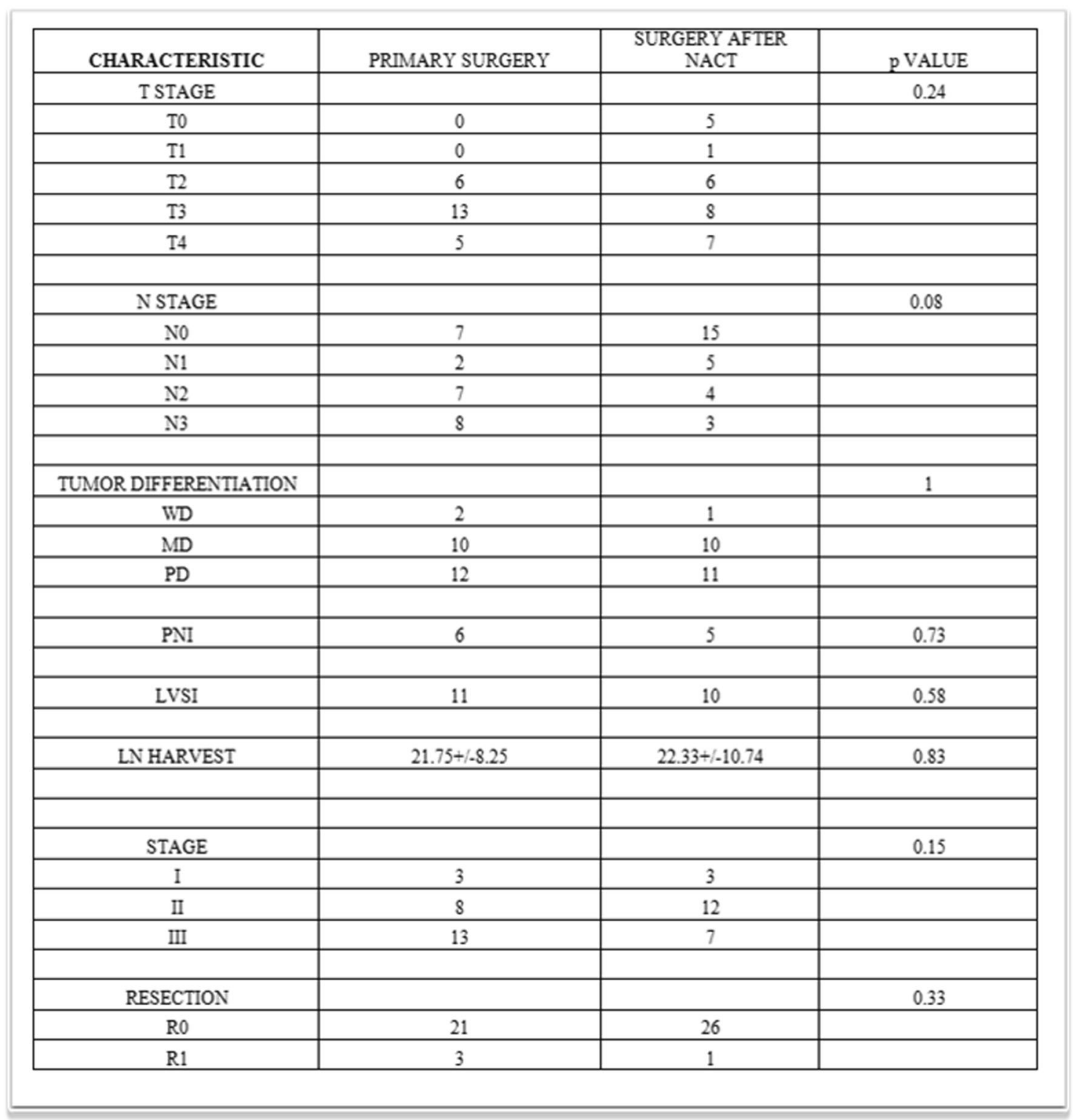

Fig. 5 Pathological characteristics 
in a significantly higher incidence of $\mathrm{R} 0$ resection compared with surgery alone (4 RCTs, $62.86 \%$ vs. $62.99 \%$, RR 1.02 , 95\% CI 0.89-1.17, $p=0.81)$.

Although resectable rate in neoadjuvant chemotherapy arm did not reach statistical significance, it was more than in upfront surgery arm. This may be because of the disappearance of small peritoneal deposits, not seen on CT. Whether this transforms to increase in overall survival is to be debated. In the present study, 5 of 27 (19\%) patients in NACT arm had a pathological complete response. We are continuously collecting survival data; our overall survival data is still not mature to answer this question.

In the present study, there was a non-significant trend for more pN0 stage in neoadjuvant chemotherapy arm compared to surgery arm $(55.6 \%$ vs. $29.2 \%, p=0.08)$. In EORTC trial, Schumacher et al. [7] reported that even though the median number of dissected lymph nodes was similar in both arms, (31 with chemotherapy; range, 5 to 80 , v 33 with surgery alone, range 10 to 88 ), lymph node metastases were more frequent in patients who had surgery alone, 52 patients (76.5\%) versus $43(61.4 \% ; p=0.018)$. In MAGIC trial, Cunningham et al. [5] reported that there was a significant trend of less advanced nodal disease (i.e., N0 or N1) in the preoperative chemotherapy group than in the surgery group $(84.4 \%$ vs. $70.5 \%, p=0.01)$. This difference may be because of the disappearance of small tumor deposits in nodes with chemotherapy. Arguing whether this transforms to increase in overall survival is to be seen by analyzing overall survival data.

In the present study, the incidence of postoperative complications was comparable between both arms. Even though there were more number of events in NACT arm, when the number of patients with grade II or more complications was taken ( $41 \%$ in primary surgery arm vs. $37 \%$ in NACT arm), it was not significant between both arms. Mean hospital stay was $8.6 \pm 1.7$ days and $9.0 \pm 1.8$ days after primary surgery and NACT arms, respectively, which was statistically not significant. There was no mortality in either of arms. Other studies have also shown no increase in postop morbidly after chemotherapy [9].

\section{Conclusions}

In patients with resectable adenocarcinoma of the stomach, neoadjuvant chemotherapy with 5-FU and Cisplatin showed a trend towards improving in the resectability rate and $\mathrm{R} 0$ resection rate. There is no increase in postoperative morbidity and mortality with neoadjuvant chemotherapy. We have to wait for survival data to answer questions whether neoadjuvant chemotherapy increases overall survival.

\section{References}

1. Dikshit RP, Mathur G, Mhatre S, Yeole BB (2011 Jan) Epidemiological review of gastric cancer in India. Indian $\mathrm{J}$ Med Paediatr Oncol 32(1):3-11

2. Mohandas KM, Jagannath P (2000) Epidemiology of digestive tract cancers in India. VI. Projected burden in the new millennium and the need for primary prevention. Indian J Gastroenterol 19(2):74-78

3. Kim JP, Lee JH, Kim SJ, Yu HJ, Yang HK (1998) Clinicopathologic characteristics and prognostic factors in 10783 patients with gastric cancer. Gastric Cancer 1(2):125-133

4. Sasako M, Sano T, Katai H, Maruyama K (1997) Radical surgery. In: Sugimura T, Sasaki MD (eds) Gastric cancer. Oxford University Press, Oxford, pp 223-248

5. Cunningham D, Allum WH, Stenning SP, Thompson JN, Van de Velde CJ et al (2006) Perioperative chemotherapy versus surgery alone for resectablegastroesophageal cancer. N Engl J Med 355(1): $11-20$

6. Y Chou M, Boige V, Pignon JP, Conroy T, Bouché O, Lebreton G, Ducourtieux M, Bedenne L, Fabre JM, Saint-Aubert B, Genève J, Lasser P, Rougier P (2011) Perioperative chemotherapy compared with surgery alone for resectablegastroesophageal adenocarcinoma: an FNCLCC and FFCD multicenter phase III trial. J Clin Oncol 29(13):1715-1721

7. Schuhmacher C, Gretschel S, Lordick F, Reichardt P, Hohenberger W, Eisenberger CF, Haag C, Mauer ME, Hasan B, Welch J, Ott K, Hoelscher A, Schneider PM, Bechstein W, Wilke H, Lutz MP, Nordlinger B, Cutsem EV, Siewert JR, Schlag PM (2010) Neoadjuvant chemotherapy compared with surgery alone for locally advanced cancer of the stomach and cardia: European Organisation for Research and Treatment of Cancer randomized trial 40954. J Clin Oncol 28:5210-5218

8. Xu AM, Huang L, Liu W, Gao S, Han WX, Wei ZJ et al (2014) Neoadjuvant chemotherapy followed by surgery versus surgery alone for gastric carcinoma: systematic review and meta-analysis of randomized controlled trials. PLoS One 9(1):e86941

9. Ott et al (2003) Neoadjuvant chemotherapy with cisplatin, 5- FU, and leucovorin (PLF) in locally advanced gastric cancer: a prospective phase II study. Gastric Cancer 6(3):159-167

Publisher's Note Springer Nature remains neutral with regard to jurisdictional claims in published maps and institutional affiliations. 